\title{
Deep Digital Flexor Contracture following Combined Tibial Plateau Levelling Osteotomy and Cranial Closing Wedge Ostectomy: A Case Report in a Dog
}

\author{
Anya Price ${ }^{1}$ Daniel Lopez ${ }^{1}$ Dominick Valenzano ${ }^{1} \quad$ Christopher Frye $^{1}$ Dro $^{-}$Ursula Krotscheck $^{1}$ \\ ${ }^{1}$ Department of Clinical Sciences, Cornell University Hospital for \\ Animals, Ithaca, New York, United States \\ Address for correspondence Daniel Lopez, DVM, Department of \\ Clinical Sciences, Cornell University College of Veterinary Medicine, \\ 930 Campus Drive, Ithaca, NY 14853, United States \\ VCOT Open 2022;5:e18-e24. \\ (e-mail: Djl242@cornell.edu).
}

\begin{abstract}
Keywords

- Z-plasty tenotomy

- dog

- deep digital flexor tendon contracture

- TPLO complication
\end{abstract}

This case report describes the use of a Z-plasty tenotomy and anastomosis to surgically lengthen the deep digital flexor (DDF) tendons of digits 2 to 5 of the pelvic limb in a 6year-old male castrated Greyhound. The procedure was used to treat contracture of this tendon complex which developed after a series of complications secondary to proximal osteotomies utilized for the treatment of cranial cruciate ligament rupture. The dog was evaluated for lameness associated with left cranial cruciate ligament rupture and excessive tibial plateau slope; accordingly, a combined tibial plateau levelling osteotomy and cranial closing wedge ostectomy was performed. Postoperatively, the dog developed substantial DDF tendon contracture that led to severe digital hyperflexion and contributed to a non-weight bearing lameness. The dog received intensive rehabilitation therapy but failed to substantially improve; therefore, all of the weight-bearing left hindlimb DDF tendons were lengthened with a Z-plasty tenotomy and anastomosis followed by further rehabilitation. Follow-up evaluation 44 months postoperatively documented mild, intermittent left hindlimb lameness on gait observation and confirmed success of the Z-plasty procedure via ultrasonographic evaluation. Conservative treatment alone was unsuccessful in managing DDF tendon contracture in this dog. Z-plasty tenotomy and anastomosis of the DDF tendon allowed for return-to-acceptable function in this case.

\section{Introduction}

Although contracture of the digital flexor tendons has been reported in small animals, it is an unusual complication after orthopaedic surgery with few reported outcomes. ${ }^{1-7}$ Contracture can lead to variable degrees of lameness. ${ }^{8,9}$ Previously reported surgical treatments for digital flexor tendon contracture include onychectomy (if isolated to one or two digits), tenectomy or tendon lengthening via tenotomy. ${ }^{1-6}$ Z-plasty tenotomy and anastomosis of the forelimb

received

November 26, 2020

accepted after revision

October 19, 2021
DOI https://doi.org/

$10.1055 / \mathrm{s}-0042-1742469$.

ISSN $2625-2325$. superficial digital flexor (SDF) tendons have been reported in the dog. ${ }^{1,6}$ To the authors' knowledge, this technique has not been reported for lengthening of the deep digital flexor (DDF) tendons. ${ }^{1,6}$

\section{Case Description}

A 6-year-old, $38.5 \mathrm{~kg}$ male castrated non-racing Greyhound was evaluated at the Cornell University Hospital for Animals in

(C) 2022. The Author(s).

This is an open access article published by Thieme under the terms of the Creative Commons Attribution License, permitting unrestricted use, distribution, and reproduction so long as the original work is properly cited. (https://creativecommons.org/licenses/by/4.0/)

Georg Thieme Verlag KG, Rüdigerstraße 14, 70469 Stuttgart, Germany 

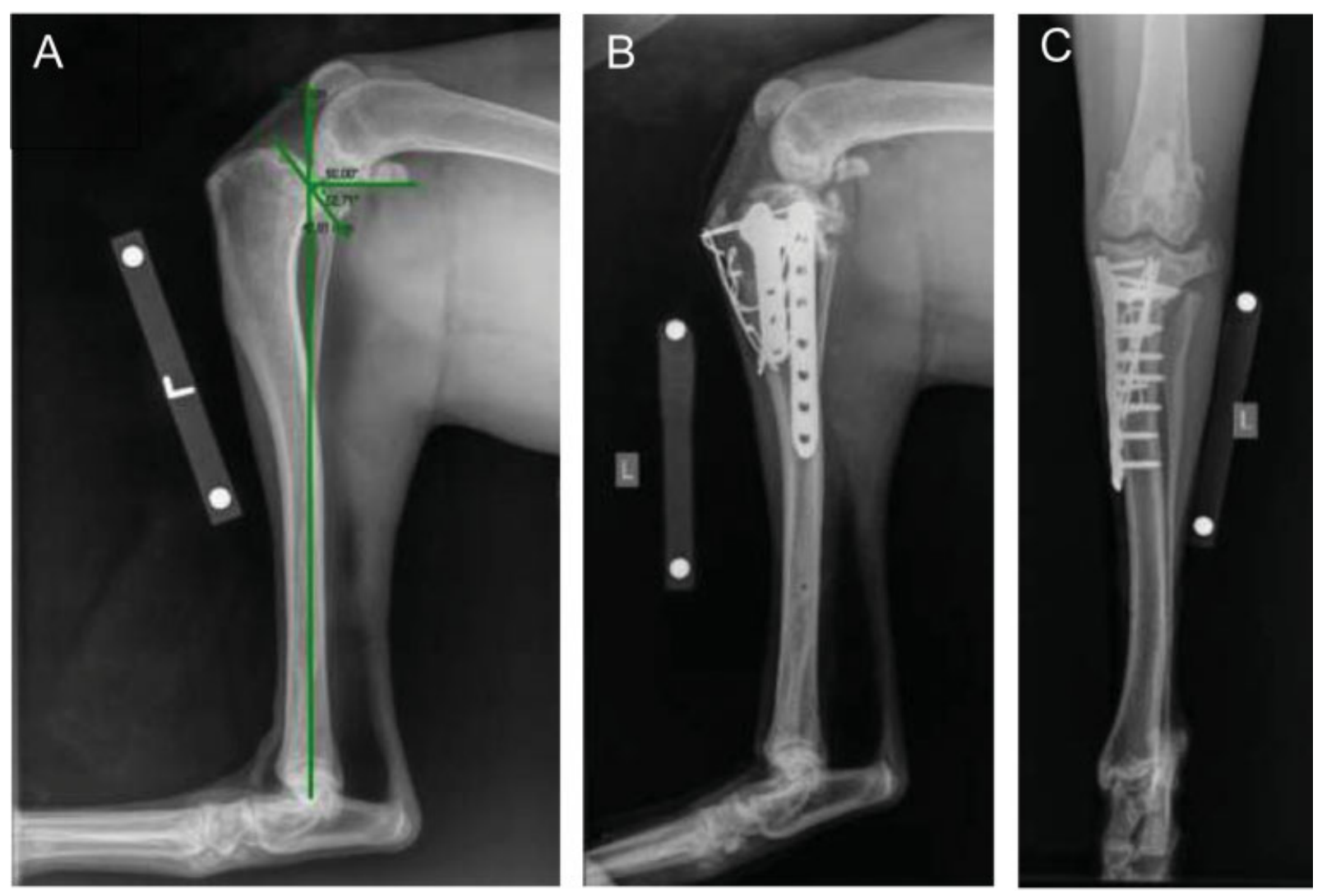

Fig. 1 (A) Preoperative mediolateral radiograph demonstrating excessive tibial slope of 53 degrees. (B) and (C) Postoperative mediolateral and craniocaudal radiograph demonstrating multiple plate fixation and pin and tension band secondary to tibial plateau levelling osteotomy and closing wedge ostectomy.

2017 for presumptive cranial cruciate ligament disease of the left stifle. Preoperative radiographs revealed an excessive tibial slope with estimated tibial plateau angle (TPA) of 53 degrees ( - Fig. 1A). Tibial plateau levelling osteotomy (TPLO) in conjunction with a cranial closing wedge ostectomy was performed using a $3.5 \mathrm{~mm}$ Synthes TPLO plate (Depuy Synthes, Warsaw, Indiana, United States), a $3.5 \mathrm{~mm}$ linear locking compression plate, ${ }^{1}$ and cranially placed tension bands. ${ }^{10}$ Arthrotomy revealed an intact meniscus. Postoperative radiographs showed a TPA of 17 degrees and an iatrogenic fibula fracture (- Fig. 1B, C). Several hours postoperatively, the dog became anxious and hyperthermic at $42.2^{\circ} \mathrm{C}$. The hyperthermia resolved with active cooling efforts and anxiolytics.

During the postoperative period, excessive bruising and serosanguinous discharge were noted at the incision site, potentially secondary to Greyhound hyperfibrinolytic syndrome. ${ }^{11}$ A modified Robert Jones bandage was placed for 10 days. After bandage removal, a mild degree of digit hyperflexion was noted which was attributed to prolonged bandaging. Passive range of motion of the digits and rehabilitation therapy were recommended.

Three weeks after surgery, the patient was diagnosed with an incisional infection and surgical site dehiscence with plate exposure. The incision cultured Escherichia coli. Doxycycline $(4.2 \mathrm{mg} / \mathrm{kg}$ orally every 12 hours) and cephalexin $(22 \mathrm{mg} / \mathrm{kg}$ orally every 12 hours) were started empirically. Topical application of a medical honey and calcium alginate dressing
(Medihoney, Integra LifeSciences Corp., Princeton, New Jersey, United States) was used for local wound management. The dog was hospitalized for 2 weeks with a modified Robert Jones bandage and honey dressing. During this time, the hyperflexion improved, and significant granulation tissue and plate coverage were noted the day of discharge. Culture results indicated sensitivity to amikacin, chloramphenicol and trimethoprim sulfamethoxazole. Doxycycline and cephalexin were continued despite culture sensitivity results due to the clinical improvement of the surgical wound. At re-examination 1 week later, the dog was weight bearing on the operated limb with no signs of knuckling or digital contracture and resolution of the incisional dehiscence. The bandage was removed. At re-examination 10 weeks postoperatively, severe atrophy of the left cranial tibial and gastrocnemius muscles was noted along with severe hyperflexion of the digits, specifically the distal interphalangeal joint, leading to a knuckled gait. No cranial tibial thrust of the left stifle was present. These findings raised suspicion for a severe contracture of the DDF tendons, and the dog was hospitalized for rehabilitation. The dog was fitted for a hindlimb Dorsi-Flex brace to allow for continued digital extension (Thera-Paw Dorsi-Flex Assist, Thera-Paw, Lebanon, New Jersey, United States). During this time, the dog developed a subcutaneous fluid pocket and a draining tract at the distal aspect of the surgical incision. The fluid cultured positive for Escherichia coli with similar sensitivity results as the previous culture. Antibiotic therapy was 
changed to trimethoprim sulfamethoxazole $(27 \mathrm{mg} / \mathrm{kg}$ orally every 12 hours) based on susceptibility results. After 1 week of inpatient rehabilitation therapy, the dog showed no improvement, characterized by inability to use the limb, knuckling of the digits and secondary right iliopsoas pain. Implant removal was recommended given the lack of clinical improvement after starting antimicrobial therapy and medical management (in comparison to the rapid improvement witnessed during the initial episode) and the marked increase in severity of digital flexor contracture (compared with the initial episode).

Radiographs 13 weeks postoperatively revealed a healed osteotomy site, although bone density was suboptimal, likely due to decreased weight bearing. Implant removal was attempted 13 weeks after the initial surgery under general anaesthesia with a prolonged femoral nerve block $(8 \mathrm{~mL} 0.5 \%$ bupivacaine, $8 \mu \mathrm{g}$ dexmedetomidine, $1.5 \mathrm{~mL}$ sarapin and $6 \mathrm{mg}$ triamcinolone). Immediate postoperative radiographs after implant removal demonstrated incomplete healing of the ostectomy site, which was likely obscured by the previous implants. A new $3.5 \mathrm{~mm}$ TPLO plate (Depuy Synthes, Warsaw, Indiana, United States) was applied to provide continued stability during healing. A Kirschner pin and tension band were left in place for additional support to the previous wedge ostectomy site ( - Fig. 2 ).

Following revision surgery and local nerve block, improved weight bearing and comfort were noted. Intensive physical therapy on the digits was performed, along with range of motion exercises and use of the hindlimb brace. However, the clinical improvement noted was transient. A progressive decrease in weight bearing, pain on digit manipulation and persistent contracture of the digits was noted
(-Video 1). Specifically, digit 2 continued to contract with underlapping in relation to digit 3 (-Fig. 3). Given these findings, surgical lengthening of the DDF tendons was recommended. A Z-plasty tenotomy and anastomosis of the DDF tendons were performed 14 weeks after the initial TPLO surgery.

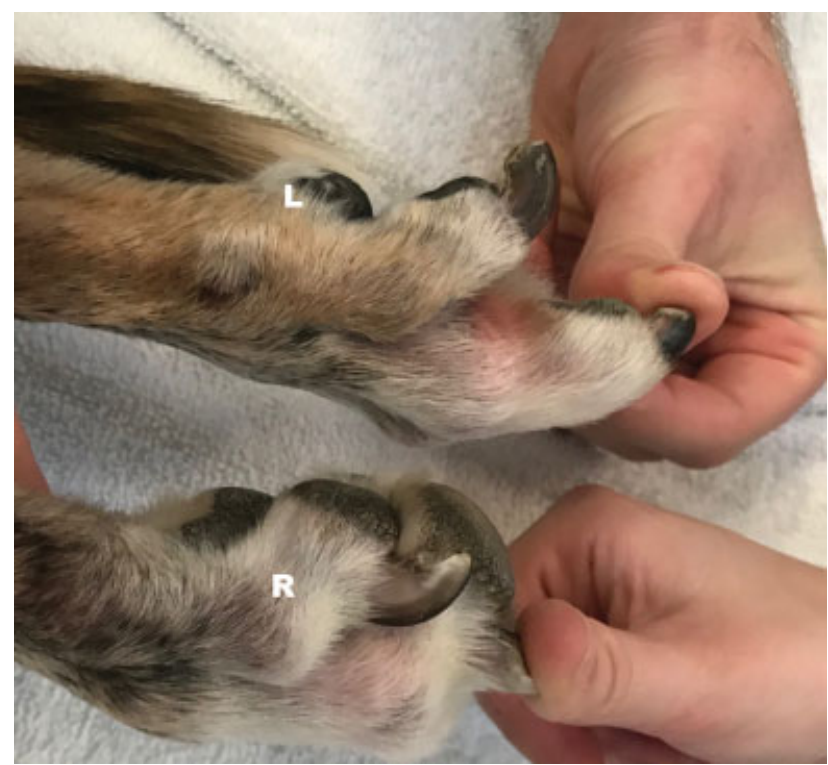

Fig. 3 Comparison between the ability of P2/P3 extension of the unaffected right (labelled R) and affected left (labelled L) pelvic limb digits prior to Z-plasty surgery.
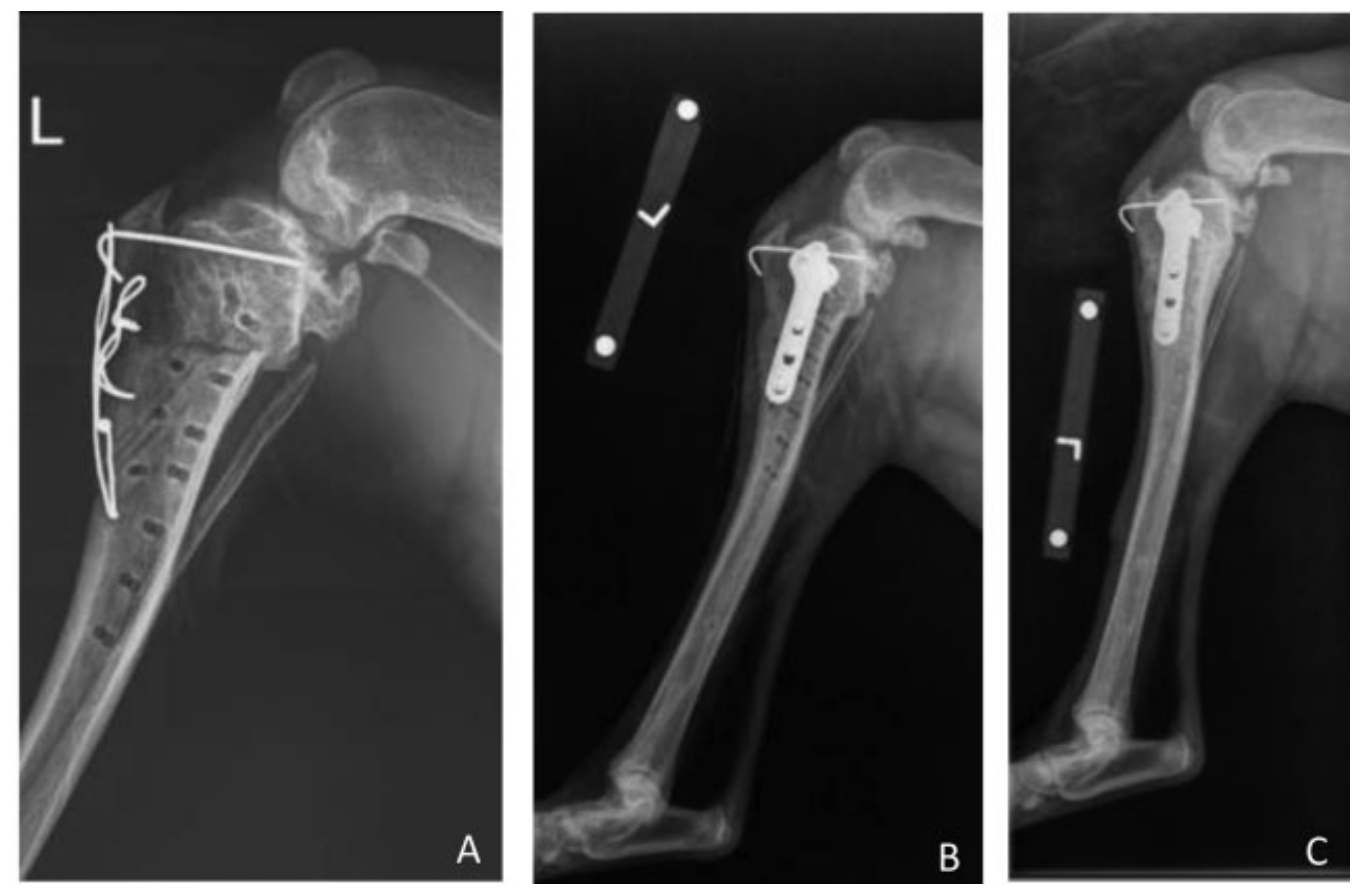

Fig. 2 (A) Intraoperative radiograph after majority of implant removal. The closing wedge ostectomy line remained radiolucent. (B) Postoperative radiograph after replacement of a tibial plateau levelling osteotomy plate over the closing wedge ostectomy. (C) Eventual complete healing of the tibial plateau levelling osteotomy and closing wedge ostectomy, 5.5 months after revision surgery. 


\section{Video 1}

Video demonstrating the lameness observed after implant revision surgery 13 weeks postoperatively, but prior to tendon lengthening procedure. Online content including video sequences viewable at: https://www.thieme-connect.com/products/ ejournals/html/10.1055/s-0042-1742469.

\section{Surgery Report}

An ultrasound-guided sciatic block was delivered using $3 \mathrm{~mL}$ $0.5 \%$ bupivacaine and $3 \mathrm{mg}$ dexmedetomidine. A psoas compartment block was delivered using $3 \mathrm{~mL} \mathrm{0.5 \%} \mathrm{bupivacaine}$ and $10 \mathrm{mg}$ dexmedetomidine. An incision was made along the plantar surface of the distal third of the metatarsal bones. The SDF tendons were mildly taut. The SDF tendons were separated to identify the DDF tendons ( $\sim$ Fig. 4A) which were

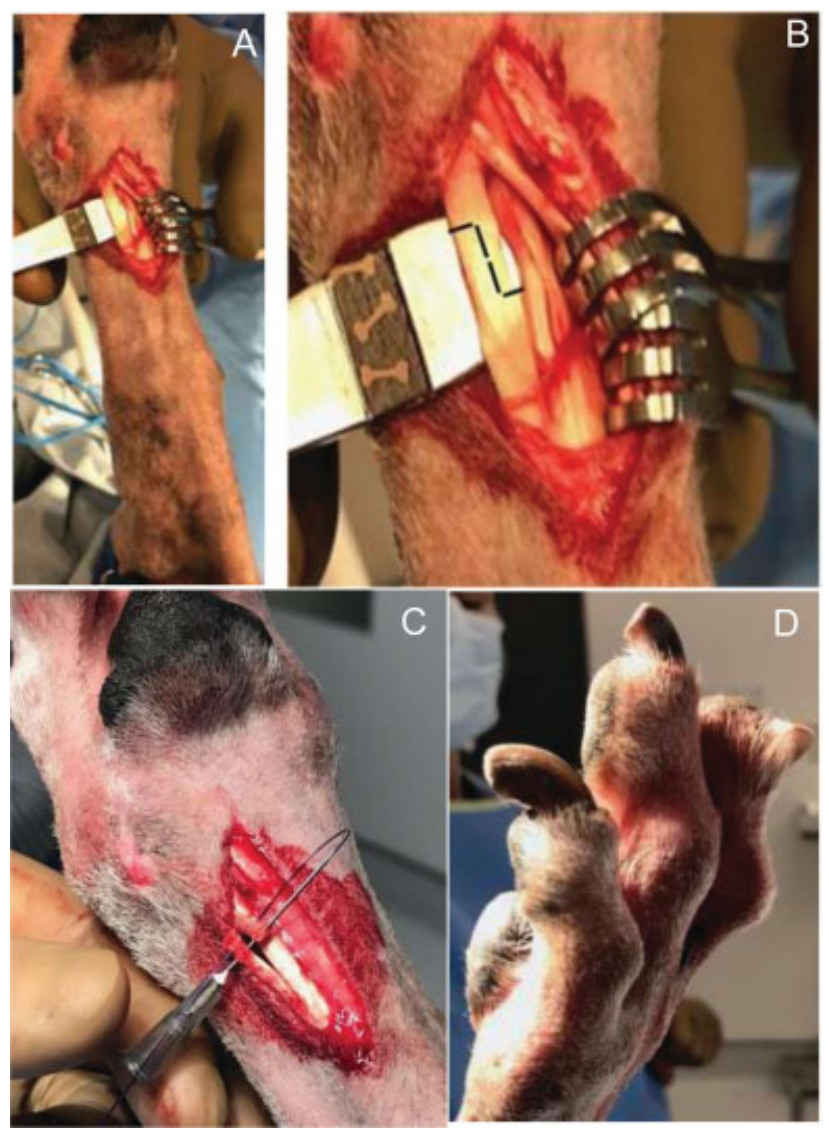

Fig. 4 (A) Isolation of the deep digital flexor tendon. (B) Depiction of the Z-plasty incision created in the deep digital flexor tendon to allow for tendon lengthening and side to side anastomosis. (C) Sideto-side anastomosis of the Z-plasty incision in the deep digital flexor tendon. Due to the extent of lengthening, the 'top' and 'bottom' portions of the Z-shaped incision are not readily visible. (D) Intraoperative view of the digits. The far two digits had deep digital flexor tendon lengthening. The near two digits have yet to be lengthened. severely taut. Each tendon was transected with an elongated Z-shaped incision ( $\mathbf{F i g}$. 4B). The initial incision within the tendon was orientated longitudinally, parallel to the long axis of the tendon. The tenotomy was completed proximally and distally by incising perpendicular to the initial tendon incision, creating a Z-shape. The tendons were allowed to lengthen and were sutured in side-to-side anastomoses with 3-0 polypropylene (-Fig. $\mathbf{4 C}$ ). The incision was closed routinely.

\section{Outcome}

The dog was managed postoperatively in a caudal splint and bandage (changed daily) to mitigate weight bearing, protect the tendon repair and maintain extension of the flexor tendons. After 8 days, the bandage was removed due to paw pad abrasions. The dog continued to bear weight on the left hindlimb without significant digital hyperflexion while in the hospital (-Video 2). The dog was discharged 2 weeks after tendon surgery with recommendations for rehabilitation and physiotherapy at home, including passive stretching of the digits in dorsiflexion and exercises aimed at low impact incremental loading of a healing tendon. At re-examination 4 weeks postoperatively, a mild weight bearing lameness was noted with mild hyperextension of the distal interphalangeal joint. The dog began to bear full weight on the left hindlimb 25 weeks after the initial surgery, with no evidence of continued DDF tendon contracture. Surgical implants were removed 40 weeks after the initial surgery due to acute incisional drainage associated with the implant; however, bacterial cultures were negative. Radiographs at that time revealed no progression of osteoarthritis. Final evaluation was performed 44 months postoperatively, consisting of clinical examination and gait observation (walk/trot) as well ultrasonographic tendon assessment. A grade $2 / 5$ lameness was identified on gait analysis ( - Video 3 ). ${ }^{12}$ No cranial tibial thrust was identified during awake examination. Full extension of the digits of the left hindlimb caused mild discomfort; however, no difference in digit position was identified during stance examination. Ultrasound evaluation documented both appropriate continuity and function of the DDF tendons, supporting successful Z-plasty anastomosis (-Fig. 5, - Videos 4 and 5). A mild left hindlimb deep digital tendinopathy was noted.

\section{Video 2}

Video demonstrating the relatively immediate and persistent improvement in lameness noted after the tendon lengthening procedure. Online content including video sequences viewable at: https://www.thiemeconnect.com/products/ejournals/html/10.1055/s0042-1742469. 


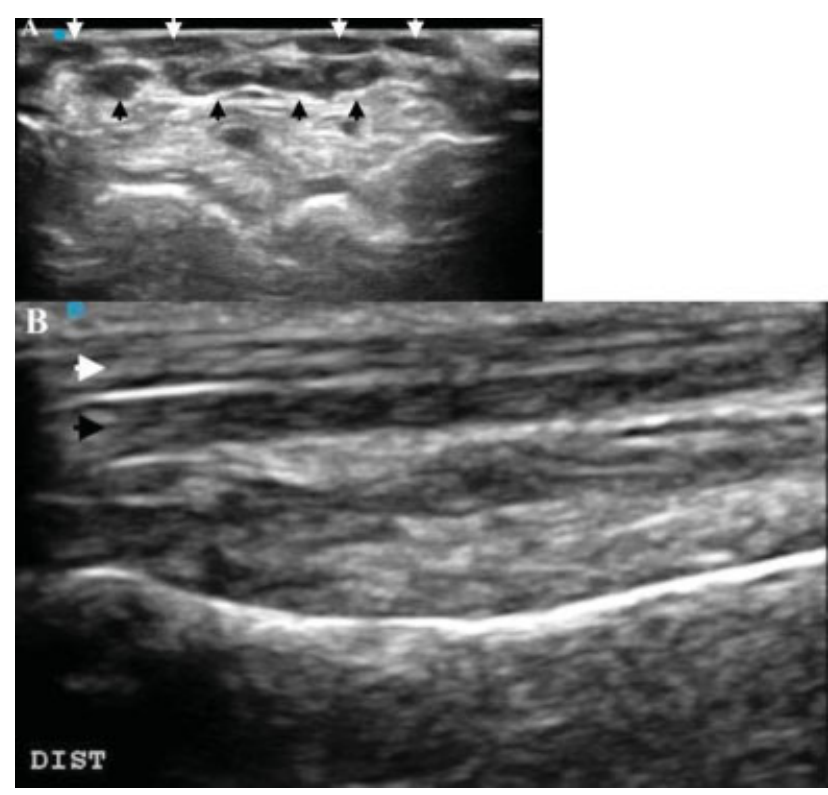

Fig. 5 (A) Representative ultrasonographic image of the left hindlimb superficial (white arrowheads) and deep (black arrowheads) digital flexor tendons in short axis. Medial is positioned to the left of the image, closest to the figure letter. (B) Representative ultrasonographic image of the left hindlimb superficial (white arrowhead) and deep (black arrowheads) digital flexor tendon of the 4th digit in long axis. Distal is located to the left of the image, closest to the figure letter. All left hindlimb deep digital flexor tendons demonstrated both static and dynamic continuity throughout their length, with no evidence of anastomotic failure. A mild deep digital flexor tendinopathy was noted characterized by mild enlargement of the deep digital flexor tendons within the metatarsal region with some thinning and mottling of appearance at the Z-plasty sites.

\section{Video 3}

Video demonstrating the mild left hindlimb lameness that was identified on long-term follow-up 44 months postoperatively. Online content including video sequences viewable at: https://www.thieme-connect. com/products/ejournals/html/10.1055/s-0042-

1742469.

\section{Video 4}

A dynamic ultrasound video identifying the superficial and deep digital flexor tendons from proximal to distal in short axis. Medial is to the left of the image. Online content including video sequences viewable at: https://www.thieme-connect.com/products/ ejournals/html/10.1055/s-0042-1742469.

\section{Video 5}

A dynamic ultrasound video evaluating the continuity and function of the superficial and deep digital flexor tendon of the third digit from proximal to distal in long axis. Distal is to the left of the image. Similar dynamic studies were acquired for all digits to evaluate the outcome of the Z-plasty procedure. Online content including video sequences viewable at: https://www.thieme-connect.com/products/ ejournals/html/10.1055/s-0042-1742469.

\section{Discussion}

This case report describes an unusual series of complications following corrective orthopaedic surgery for cranial cruciate ligament insufficiency, with subsequent contracture of the left hindlimb DDF tendons leading to impaired clinical function necessitating tenoplasty.

The DDF tendon, responsible for flexion of the hindlimb digits, originates on the caudal surface of the proximal fibula, the proximal caudolateral border of the tibia and the interosseous membrane. At the level of the metatarsus, it divides into four individual tendons, which all pass through the manica flexoria to insert on the flexor tubercles of the distal phalanges. ${ }^{13}$ Abnormalities at any level of the musculotendinous unit can lead to contracture. Causes of tendon contracture include congenital anomalies, parasitism, dietary imbalances, improper environment, hyperthermia or trauma. $2,3,6,7,14,15$ With regard to this dog, the cause of contracture remains elusive; however, the chain of postoperative complications all likely contributed to progressive contracture. Trauma and inflammation from the initial osteotomies, the iatrogenic fibular fracture or the persistent implant infection may have damaged the musculotendinous unit of the DDF tendon and contributed to contracture. The dog's postoperative hyperthermia may also have been an inciting cause for contracture. ${ }^{16}$ In addition, quadriceps contracture is reported after prolonged immobilization following femur fracture. ${ }^{9,17}$ It is possible that in this case, DDF contracture may have been a consequence of the restricted motion from bandaging, especially given sighthound susceptibility to soft tissue injury from external coaptation. ${ }^{18}$ Ideally, prophylactic treatment with aminocaproic acid could have been considered. ${ }^{19}$ Finally, an ultrasound evaluation of the musculotendinous unit upon initial diagnosis would have been useful for characterizing the injury further, specifically with regard to myositis.

Treatment of tendinous contractures can be divided into medical and surgical management. Medical management includes continuous splintage in tension, physiotherapy or reduced activity. ${ }^{1,20}$ Surgical intervention has only been 
recommended in severe or chronic cases. ${ }^{17}$ Surgical treatments for tendinous contracture include a tenotomy/tenectomy or a tendon lengthening procedure. Cats with digit contracture secondary to onychectomy have been treated with tenectomy of the DDF and SDF for rapid resolution of lameness. ${ }^{2,3}$ While a tenotomy/tenectomy provides rapid correction of contracture, it is not desirable in every case due to the loss of function of the muscle-tendon unit. Most concerning about DDF tendon transection is the hyperextension of $\mathrm{P} 3$, resulting in the digits remaining permanently off the ground and caudal displacement in weight bearing forces. ${ }^{21}$ In this dog, a tendon-lengthening procedure was elected over tenotomy/tenectomy due to contracture affecting all four digits and the desire to preserve function of the muscle-tendon unit. A Z-plasty tenotomy and anastomosis were elected given previous reports of SDF tendon lengthening in a Great Dane as well as lengthening of both the SDF and flexor carpi ulnaris tendon in an Akita. ${ }^{1,6}$ This procedure has been utilized successfully in humans to regain proper weight bearing and functional activity after orthopaedic trauma. ${ }^{15,22}$

During the recovery period post Z-plasty procedure, bandage sores led to the premature removal of external coaptation. The additional forces experienced at the level of the tendon repair likely placed the anastomosis at increased risk of failure, given the timeline of normal tendon healing. ${ }^{16,23}$ The decision to prematurely remove the bandage was based on the increasing severity of bandage sores outweighing the potential for anastomosis failure. Despite the premature removal of external coaptation, clinical examination demonstrated adequate tendon function and ultrasonographic evaluation demonstrated both static and dynamic continuity of all previously lengthened tendons supporting success of the Z-plasty procedure. Ultimately, a mild lameness persisted which could be secondary to expected progression of osteoarthritis, the mild DDF tendinopathy identified during ultrasonographic evaluation or other causes such as persistent dynamic instability of the left stifle not detected on awake examination. ${ }^{24,25}$ Radiographic evaluation at final follow-up was not performed but would have allowed for evaluation of osteoarthritis progression.

A limitation of this case report revolves around the lack of ultrasonographic information of the musculotendinous unit of the DDF tendon in the initial postoperative period. Identification of a reversible pathology secondary to the numerous complications encountered affecting the DDF tendon complex may have nullified the need for surgical intervention in this case. However, surgical intervention of the tendon contracture in this specific case allowed for rapid return of function.

\section{Conclusion}

This case report describes a series of complications following proximal tibial osteotomies resulting in DDF contracture. The contracture and associated lameness were successfully treated with tenoplasty after unsuccessful medical manage- ment. Long-term outcome of this case was satisfactory based on follow-up orthopaedic examination and tendon ultrasonographic evaluation.

\section{Authors' Contribution}

AP and DL conceptualized and designed the study, acquired the data and prepared the manuscript.

$\mathrm{DV}, \mathrm{CF}$, and UK conceptualized on the study and prepared the manuscript.

\section{Funding}

None.

\section{Conflict of Interest}

The authors have no conflict of interest and are responsible for the writing and the content of this paper.

\section{Acknowledgments}

We thank Dr Kei Hayashi and Dr Rory Todhunter for their contributions to patient management.

\section{References}

1 Bailey CJ, Culvenor JA. The surgical management of superficial digital flexor contracture in a Great Dane. Aust Vet J 2007;85(12):20-22

2 Cabon Q Plante J, Gatineau M. Digital flexor tendon contracture treated by tenectomy: different clinical presentations in three cats. JFMS Open Rep 2015;1(02):2055116915597237

3 Cooper MA, Laverty PH, Soiderer EE. Bilateral flexor tendon contracture following onychectomy in 2 cats. Can Vet J 2005;46 (03):244-246

4 Thom LK, Pool RR Jr, Malik R. Digital flexor musculotendinous contracture in two Devon Rex cats. J Feline Med Surg 2017;19(03): 304-310

5 Towle H, Friedlander K, Ko R, Aper R, Breur G. Surgical treatment of simple syndactylism with secondary deep digital flexor tendon contracture in a Basset Hound. Vet Comp Orthop Traumatol 2007; 20(03):219-223

6 Gondolfe M, Garneau M. Correction of carpal valgus and flexural deformity with z-tenotomy and anastomosis procedure in a dog. Vet Comp Orthop Traumatol 2020;3:28-32

7 Devor M, Sørby R. Fibrotic contracture of the canine infraspinatus muscle: pathophysiology and prevention by early surgical intervention. Vet Comp Orthop Traumatol 2006;19(02):117-121

8 Leighton RL. Muscle contractures in the limbs of dogs and cats. Vet Surg 1981;10:132-135

9 Taylor J, Tangner CH. Acquired muscle contractures in the dog and cat. A review of the literature and case report. Vet Comp Orthop Traumatol 2007;20(02):79-85

10 Talaat MB, Kowaleski MP, Boudrieau RJ. Combination tibial plateau leveling osteotomy and cranial closing wedge osteotomy of the tibia for the treatment of cranial cruciate ligament-deficient stifles with excessive tibial plateau angle. Vet Surg 2006;35(08): 729-739

11 Lara-García A, Couto CG, Iazbik MC, Brooks MB. Postoperative bleeding in retired racing greyhounds. J Vet Intern Med 2008;22 (03):525-533

12 McCarthy G, O’Donovan J, Jones B, McAllister H, Seed M, Mooney C. Randomised double-blind, positive-controlled trial to assess the efficacy of glucosamine/chondroitin sulfate for the treatment of dogs with osteoarthritis. Vet J 2007;174(01):54-61

13 Hermanson J. The muscular system. In: Miller and Evans' Anatomy of the Dog. 5th edition. St. Louis: Elsevier; 2020:309-311 
14 Woo SL, Gelberman RH, Cobb NG, Amiel D, Lothringer K, Akeson WH. The importance of controlled passive mobilization on flexor tendon healing. A biomechanical study. Acta Orthop Scand 1981; 52(06):615-622

15 Sharma G, Makwana M, Guha A. Extensor hallucis longus tendon contracture following triplane fracture of the tibia. Foot Ankle Surg 2007;13:76-79

16 Sharma P, Mafulli N. Biology of tendon healing. J Musculoskelet Neuronal Interact 2006;6:181-190

17 Bloomberg M. Muscles and tendons. In: Slatter D, , ed. Textbook of Small Animal Surgery. Philadelphia: WB Saunders; 1993:1996-2019

18 Meeson RL, Davidson C, Arthurs GI. Soft-tissue injuries associated with cast application for distal limb orthopaedic conditions. A retrospective study of sixty dogs and cats. Vet Comp Orthop Traumatol 2011;24(02):126-131

19 Marín LM, Iazbik MC, Zaldivar-Lopez S, Guillaumin J, McLoughlin MA, Couto CG. Epsilon aminocaproic acid for the prevention of delayed postoperative bleeding in retired racing greyhounds undergoing gonadectomy. Vet Surg 2012;41(05):594-603
20 Carmichael S, Marshall W. Muscle and tendon disorders. In: Veterinary Surgery: Small Animal. 2nd edition St. Louis: Elsevier; 2018:1318-1320

21 Moores A. Muscle and tendon disorders in small animals 2. Conditions affecting the hindlimb and digital flexor tendons. In Pract 2012;34:74-77

22 Fitoussi F, Bachy M. Tendon lengthening and transfer. Orthop Traumatol Surg Res 2015;101(1, Suppl):S149-S157

23 Dueland R, Quenin J. Triceps tenotomy: biomechanical assessment of healing strength. J Am Anim Hosp Assoc 1980;16:507

24 Innes JF, Costello M, Barr FJ, Rudorf H, Barr ARS. Radiographic progression of osteoarthritis of the canine stifle joint: a prospective study. Vet Radiol Ultrasound 2004;45(02): 143-148

25 Shimada M, Mizokami N, Ichinohe T, et al. Long-term outcome and progression of osteoarthritis in uncomplicated cases of cranial cruciate ligament rupture treated by tibial plateau leveling osteotomy in dogs. J Vet Med Sci 2020;82(07): 908-916 